\title{
Evaluation of drought tolerance in corn (Zea mays L.) new hybrids with using stress tolerance indices
}

\author{
H. Moradi ${ }^{1}$, G A. Akbari ${ }^{2}$, S. Khavari Khorasani ${ }^{3}$, H A. Ramshini ${ }^{4}$
}

\begin{abstract}
In order to study the effect of drought stress on morphophysiologic characteristics, yield and yield components of 8 new hybrids of corn (Zea maize L.) and KSC704 commercial hybrid as control resistant to drought and warm (which were bred and screened in Khozestan province condition), an experiment was conducted in a Randomized Complete Block Design (RCBD) with three replications under drought stress and normal irrigation at Khorasan-Razavi Agriculture Research Center, Mashhad, Iran on June 10, 2011. The results of analyze variance showed that under normal irrigation and drought condition, there was a significant difference $(p<0.01)$ between the hybrids. Mean comparison of hybrids revealed that in normal irrigation $\mathrm{H} 6$ and in drought stress H8 hybrid had the maximum grain yield (12.85 and 6.75 ton/ha, respectively). Based on the grain yields of studied hybrids, stress tolerance index (STI), stress susceptibility index (SSI), tolerance index (TOI), mean productivity (MP), geometric mean productivity (GMP), harmonic mean (HM) and golden mean (GM) were estimated. Results showed that among drought tolerance indices, MP, GMP, STI and HM were the best indices for corn and KSC704 hybrid and H4 had the highest tolerance to drought in Mashhad weather condition.
\end{abstract}

Keywords: tolerance indices, drought stress, corn, yield and yield components

\section{Introduction}

According to the FAO reports (2008) in Iran (annual rainfall 24mm), the production of maize was estimated 2.8 percent of total cereals production and 1.6

${ }^{1}$ MSc student of Agronomy- Abouraihan Campus- University of Tehran- Iran .

h_moradi@ut.ac.ir

${ }^{2}$ Assistant Professor - Dept of Agronomy \& Plant Breeding- College of Agricuulture Abouraihan Campus, University of Tehran- Iran.

${ }^{3}$ Assistant Professor of Khorasan-Razavi Agriculture Research Center, Mashhad, Iran.

${ }^{4}$ Assistant Professor - Dept of Agronomy \& Plant Breeding- College of Agricuulture Abouraihan Campus, University of Tehran- Iran. 
million tons grain yield from 0.25 million hectare of arable and cultivated lands, however hybrid grain production is extremely low (Moradi Dezfuli et al., 2008). Corn has been being cultivated and grown around the world and drought pressure was classified as one of the most deleterious environmental stresses which restrict crop production (Alahdadi et al., 2011; Khodarahmpour, 2011; Oraki et al., 2011; Song et al., 2010; Sinaki et al., 2007) and 20-25 percent of the planting area of maize is affected by drought pressure in the world (Golbashy et al., 2010). Corn is used as food and feed for livestock and meets the requirements of material in different industries such as food, medicine and textile and security of food in the world relies on growth and development of plants which are highly tolerate to abiotic stresses particularly drought (Ali et al., 2011). Drought tolerance selection is not easy due to the happening of strong interactions between genotypes and the environment and restricted knowledge about the function and role of tolerance mechanisms (Messmer, 2006). Among different factors which affect and decline corn yield, 31 percent was belong to drought stress and thicker plant stand, using various hybrids, differences in irrigation methods and climatic conditions should be considered in corn yield reduction (Eck, 1984). Drought stress affects leaf water content, photosynthesis and water use efficiency (WUE) (Egilla et al., 2005). Reports showed that in semi arid regions of Iran, drought declines season length (Magorocosho et al., 2003), disturb photosynthesis and assimilate remobilization which finally reduces grain weight (Vaezi and Ahmadikhah, 2010). It has been shown that water shortage declines corn canopy height, leaf area index and root growth (Hirich et al., 2012; Payero et al., 2006). Corn yield components are controlled by many genes which react to the lack of water with different flexibility (Esmailiyan et al., 2008) but it is affected by the environmental condition, either (Farre et al., 2000). Grain yield reduction of maize due to the drought pressure is varied between 1to $76 \%$ depending on the severity, timing and stage of occurrence (Mostafavi et al., 2011; Zarabi et al., 2011; Song et al., 2010). Oktem (2008) found that under water pressure, grain yield was reduced to 37 percent because of 18 percent kernel weight reduction and 1 percent reduction in the number of kernel. Fernandez (1992) divided the reaction of corn genotypes in stress and non-stress condition into four groups: higher yield than average in both condition (A group), higher yield than average in non-stress condition (B group), higher yield than average in stress condition ( $\mathrm{C}$ group) and lower yield than average in both condition (D group) and claimed that an index can separate hybrids of A group from other is the most suitable index. Fisher and Maurer (1978) presented stress susceptibility index (SSI) and announced lower SSI, more resistance to drought. Selection based on SSI, causes selection of hybrids which have higher yield in stress condition but have lower yield in non-stress condition. Rosielli and Hamblin (1981) demonstrated that lower stress tolerance index (STI), 
hybrid yield in normal irrigation and drought condition is close to each other or plant is resistant to drought. This study was carried out in order to evaluate corn hybrids reaction to drought pressure and determine the best measures for increase and improvement of hybrids yield in stress and non-stress condition regarding the investigate the relationship of grain yield and its related traits and the diversity of quantitative traits between the studied hybrids in both conditions. Jafari et al., (2009) claimed that Stress Tolerant Index (STI) was more useful in order to select favorable corn cultivars under stressful and stress-free conditions.

\section{Material and method}

In this experiment the effect of drought stress on morphophysiologic characteristics, yield and yield components of 8 new hybrids of corn (Zea maize L.) and KSC704 commercial hybrid as control resistant to drought and warm (which were bred and screened in Khozestan province condition) was assessed at Khorasan-Razavi Agriculture Research Center, Mashhad, Iran (6 km of SouthEast of Mashhad, 3616N and 5938E, altitude $985 \mathrm{~m}$ ). Climate in this region is cool and dry with average annual precipitation of $286 \mathrm{~mm}$ and all the rain fells in autumn and winter. The experiment was planted on June 10, 200as a Randomized Complete Block Design (RCBD) with three replications. The hybrids were grown in two-row plots with $3.15 \mathrm{~m}$ length and $0.75 \mathrm{~cm}$ spacing between rows. The plant density was 7500 plant/ha. In every pile, 3 seeds were planted which after seedling establishment and emergence were reduced to 1 plant. Cultivation operations except irrigation were done according to typical practices in Research Station. After seedbed preparation, $130 \mathrm{~kg}$ ammonium phosphate and $88 \mathrm{~kg}$ urea per hectare were applied and also, $88 \mathrm{~kg}$ urea was used at 7-leaf stage top dressing. For application of irrigation treatments, based on soil test, irrigation was applied based on 50 and $80 \%$ allowing water depletion for non stress and stress conditions, respectively. During the growth season, agronomic and morphological characteristics such as ear height, length of raceme, stem diameter, leaves no. and upper leaves no. of the genotypes were measured on 10 competitive plants in each plot randomly. At harvesting time, the number of plants and ears harvested were counted separately. Yield components (ear length and diameter, cob diameter, kernel depth, row no. / ear, kernel no. / row, total kernel no./ear and 300-kernel weight were measured. After separation of kernels by schiller and determining the humidity percentage of grains by digital handy psychrometer (Dicky John model), final grain yield in each experimental plot (based on $14 \%$ humidity) was corrected and calculated at ton per hectare. stress tolerance index (STI), stress susceptibility index (SSI), tolerance index (TOL), mean productivity (MP), geometric mean productivity (GMP), harmonic mean (HARM) and golden mean (GOL) were 
calculated by below formula:

$\begin{array}{llll}\text { (1) } S T I=\frac{Y_{P} \times Y_{S}}{\left(\bar{Y}_{P}\right)^{2}} & \text { (2) } T O L=Y p-Y s & \text { (3) } G M P=\sqrt{(Y p)(Y s)} & \text { (4) } M P=\end{array}$ $\frac{Y p+Y s}{2}$

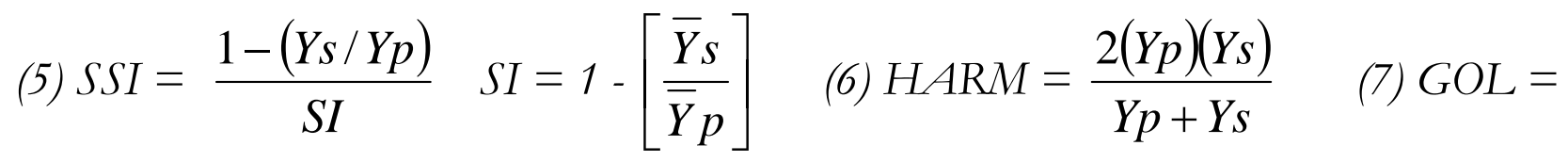
$\frac{Y p+Y s}{Y p-Y s}$

$\mathrm{Yp}=$ potential yield of genotype in non-stress condition

Ys $=$ potential yield of genotype in stress condition

$\bar{Y} \mathrm{p}=$ mean of genotypes yield in non-stress condition

$\bar{Y}$ s $=$ mean of genotypes yield in stress condition

After data collection, they were recorded by Excel software. Data analyze variation and means comparison were calculated by SAS (ver 9.1), SPSS (ver 16) statistical software and Duncan Multiple Range Test (DMRT).

\section{Results and discussion}

Analyze variance showed that in non-stress condition, except for tassel length, stem diameter, leaves no, ear/plant no, 10 ear weights, total kernels no and grain yield and in stress condition except for ear diameter, there was a significant difference between studied hybrids (table 1). In non-stress condition, H6 (12.85 ton/ha) had the highest yield, followed by KSC704 and H1 (12.55 and 12.33 ton/ha, respectively), while $\mathrm{H} 8, \mathrm{H} 4$ and control (6.75, 5.46 and 4.60 ton/ha, respectively) had the highest grain yield in drought pressure. KSC704 (96.55 gr) and H7 (78.80 gr) had the highest and lowest 300-kernel weight in normal irrigation. Maximum and minimum kernel depth was recorded for KSC704 and $\mathrm{H} 1$ (9.01 and $5.32 \mathrm{~mm}$ ) in drought tension and $\mathrm{H} 6$ and $\mathrm{H} 8 \mathrm{Had}$ the maximum and minimum ones in normal irrigation. Among the studied hybrids, H8 and H2 (10.017 and 10.050 ton/ha) had the lowest kernel yield in normal irrigation. Lower 
yield of $\mathrm{H} 8$ can be attributed to lower kernel depth and kernels number. Also, lower yield of $\mathrm{H} 2$ can be due to the length of flag leaf, 300-kernel weight and kernel no/row in comparison to the other hybrids. In drought pressure, H3 (1.99 ton/ha) had the minimum kernel yield because of row no/ear ( 9.7 row averagely) and kernel no/ear (12.01) and ear weight. Table 1 shows that drought tension considerably reduced all the studied traits. So that, the highest percentage of reduction was recorded for 10 ear weight (-65.9), total kernels no (-63.88), and kernel yield (-61.21). Lorense et al., (1987) reported that the number of kernels was the most susceptible yield components to water shortage. Ouattar et al., (1987) found that drought pressure reduced corn kernel yield which was related to the reduction of kernel no than kernel weight. In addition, these authors indicated that drought pressure declined ear length, weight and diameter. Cob weight was decreased more than $38 \%$ by drought stress. Since kernel yield depends on row no/ear, total kernel no/ear, kernel depth and 300-kernel weight, it can be deduced that kernel yield reduction was due to the cumulative effects of these yield components. According to the table 1, kernel no/row was more damaged by drought $(53.51 \%)$. The main reason is that drought pressure at flowering stage delays tasseling appearance. So tassels are appeared when pollination was done and there is no any visible pollen, ovules are not fertilized and finally kernels will not being formed. Another reason is that the embryo of fertilized ovules are aborted, kernel is not formed which lead to lower kernel no/ear and kernel no/row. Drought tension increased upper leaves no and ear/ plant no. Generally, it can be concluded that total kernel no/ear (-63.88 \%), kernel depth $(-24.54 \%)$ and 300-kernel weight $(-15.67 \%)$ reduced kernel yield and ear no/plant improved kernel yield $(28.71 \%)$. 
Table 1: Analyze variation of corn hybrids traits under stress and non-stress condition

\begin{tabular}{|c|c|c|c|c|c|c|c|c|c|c|c|}
\hline \multirow[b]{2}{*}{ Variation \% } & \multirow[b]{2}{*}{ Mean } & \multicolumn{4}{|c|}{ Drought condition } & \multicolumn{5}{|c|}{ Normal irrigation } & \multirow[b]{2}{*}{ Trait } \\
\hline & & $\mathbf{C V}$ & Error & Hybrid & Replication & Mean & $\mathbf{C V}$ & Error & Hybrid & Replication & \\
\hline-33.17 & 164.05 & 2.74 & 20.30 & $229.35 * *$ & $2669.44^{* *}$ & 245.90 & 2.48 & 37.44 & $398.83 * *$ & $40.01 \mathrm{~ns}$ & Plant height (cm) \\
\hline-38.48 & 126.27 & 3.09 & 15.30 & $218.30 * *$ & $1831.84^{* *}$ & 205.26 & 2.40 & 24.39 & $427.90^{* *}$ & 50.89ns & Flag leaf height $(\mathrm{cm})$ \\
\hline-7.03 & 37.77 & 6.19 & 5.47 & $24.87 * *$ & $78.61 * *$ & 40.63 & 8.48 & 11.89 & 21.50ns & 14.98ns & Tassel length (cm) \\
\hline-32.97 & 77.21 & 4.67 & 13.04 & $108.12 * *$ & $848.45^{* *}$ & 115.19 & 6.31 & 52.92 & $412.60 * *$ & $4.26 \mathrm{~ns}$ & Ear height (cm) \\
\hline-36.42 & 16.77 & 10.20 & 2.92 & $17.97 * *$ & $8.55 n s$ & 26.38 & 6.12 & 2.61 & $2.10 \mathrm{~ns}$ & $0.98 \mathrm{~ns}$ & Stem diameter (mm) \\
\hline-9.41 & 13.28 & 3.79 & 0.25 & $2.34 * *$ & $2.25 * *$ & 14.66 & 4.08 & 0.35 & $0.56 \mathrm{~ns}$ & $0.40 \mathrm{~ns}$ & Leaves no. \\
\hline+2.25 & 5.89 & 3.40 & 0.04 & $0.21 * *$ & $0.006 \mathrm{~ns}$ & 5.76 & 4.44 & 0.06 & $0.42 * *$ & $0.22 \mathrm{~ns}$ & Upper leaves no. \\
\hline+28.71 & 1.30 & 13.37 & 0.03 & $0.16 * *$ & $0.02 \mathrm{~ns}$ & 1.01 & 6.41 & 0.004 & $0.005 \mathrm{~ns}$ & $0.0003 \mathrm{~ns}$ & Ear no/plant \\
\hline-65.90 & 0.90 & 14.75 & 0.01 & $0.07 * *$ & $0.01 \mathrm{~ns}$ & 2.64 & 10.46 & 0.07 & $0.11 \mathrm{~ns}$ & $0.007 \mathrm{~ns}$ & 10 ear weight (kg) \\
\hline-38 & 0.31 & 13.73 & 0.001 & $0.01 * *$ & $0.0005 \mathrm{~ns}$ & 0.50 & 13.03 & 0.004 & $0.15 *$ & $0.002 \mathrm{~ns}$ & 10 cob weight (kg) \\
\hline-15.67 & 73.45 & 5.02 & 13.62 & $209.68 * *$ & $215.94^{* *}$ & 87.10 & 7.88 & 47.18 & $130.46^{*}$ & $69.04 \mathrm{~ns}$ & 300-kernel weight (gr) \\
\hline-24.34 & 11.90 & 7.84 & 0.87 & $4.61 * *$ & $0.26 \mathrm{~ns}$ & 15.73 & 5.17 & 0.66 & $2.36 *$ & $0.23 \mathrm{~ns}$ & Row no/ear \\
\hline-53.51 & 18.45 & 14.57 & 7.23 & $31.78^{* *}$ & $2.18 \mathrm{~ns}$ & 39.69 & 5.19 & 4.24 & $28.84^{* *}$ & $0.16 \mathrm{~ns}$ & Kernel no/row \\
\hline-63.88 & 224.86 & 20.75 & 2178.87 & $9720.86^{* *}$ & 897.36ns & 622.64 & 6.61 & 1696.72 & 4026.04ns & $1385.55 \mathrm{~ns}$ & Total kernel no/ear \\
\hline-34.54 & 12.41 & 7.83 & 0.94 & $5.84 * *$ & $3.37 \mathrm{~ns}$ & 18.96 & 4.64 & 0.77 & $5.73 * *$ & $1.52 \mathrm{~ns}$ & Ear length (cm) \\
\hline-26.18 & 36.50 & 14.30 & 27.25 & 43.95ns & $12.41 \mathrm{~ns}$ & 49.45 & 3.82 & 3.58 & $12.51^{*}$ & $0.54 \mathrm{~ns}$ & Ear diameter (mm) \\
\hline-25.55 & 22.81 & 14.19 & 10.49 & $34.02 *$ & $2.51 \mathrm{~ns}$ & 30.64 & 4.74 & 2.11 & $9.83 * *$ & $0.45 \mathrm{~ns}$ & Cob diameter (mm) \\
\hline-24.54 & 7.10 & 12.31 & 0.76 & $3.73 * *$ & $0.03 \mathrm{~ns}$ & 9.41 & 6.09 & 0.32 & $1.17^{*}$ & $0.23 \mathrm{~ns}$ & Kernel depth (mm) \\
\hline-20.98 & 0.64 & 6.49 & 0.001 & $0.01 * *$ & $0.003 \mathrm{~ns}$ & 0.81 & 1.41 & 0.0003 & $0.001 * *$ & $0.0005 n s$ & Kernel percentage \\
\hline-61.21 & 4.27 & 18.68 & 0.63 & $5.23 * *$ & $0.76 \mathrm{~ns}$ & 11.01 & 12.88 & 2.01 & $4.24 \mathrm{~ns}$ & $0.58 \mathrm{~ns}$ & Total yield (ton/ha) \\
\hline
\end{tabular}

**: Significant at 1\%; *: Significant at 5\%; ns: non significant 
In order to investigate phenotypic correlation of studied traits with each other and yield, simple correlation analysis was done and the results were presented in tables 2 and 3 . Under non-stress condition, kernel yield had a positive and significant correlation with plant height, 300-kernel weight, flag leaf height, kernel no/row and total kernel no/ear and there was not any statistic significant difference between other traits and kernel yield (table 2). Kernel yield had the highest positive correlation with plant height (0.84) and 300-kernel weight (0.83) which was in line with the findings of Bolanos and Edemedes (1996). Dash et al., (1999) reported there was a correlation between kernel yield and kernel no/row and ear length. The results of phenotypic correlation analysis between other traits in normal irrigation showed that plant height and flag leaf height had the highest positive and significant correlation (0.97), followed by flag leaf height and ear height (0.94) and maximum negative correlation was recorded between cob diameter and kernel percentage (-0.86) (table 2). Furthermore, in drought tension, had a positive and significant correlation with kernel no/row, ear no/plant and ear length. Grain yield had a negative and significant correlation with upper leaves no (-0.68). Ear length are controlled by genotypic and environmental factors such as nutrients and water content. Since ear contains kernels and has been being reported as an important part of yield, so, more ear length, more kernels which leads to higher yield. It was reported that there was a correlation between grain yield and ear dimension $(\mathrm{r}=0.751)$, ear weight $(\mathrm{r}=0.548)$, kernel no per ear $(\mathrm{r}=$ 0.646), kernel weight ear $(r=0.426)$ and 1000-grain weight $(r=0.552)$ (Palta et al., 2011; Khazaei et al., 2010). Kernel no/row and total kernel no/ear had the maximum phenotypic correlation (0.97), followed by plant height and flag leaf height (0.94). Also, ear no/plant and upper leaves no had the maximum negative and significant correlation (-0.85) (table 3). 


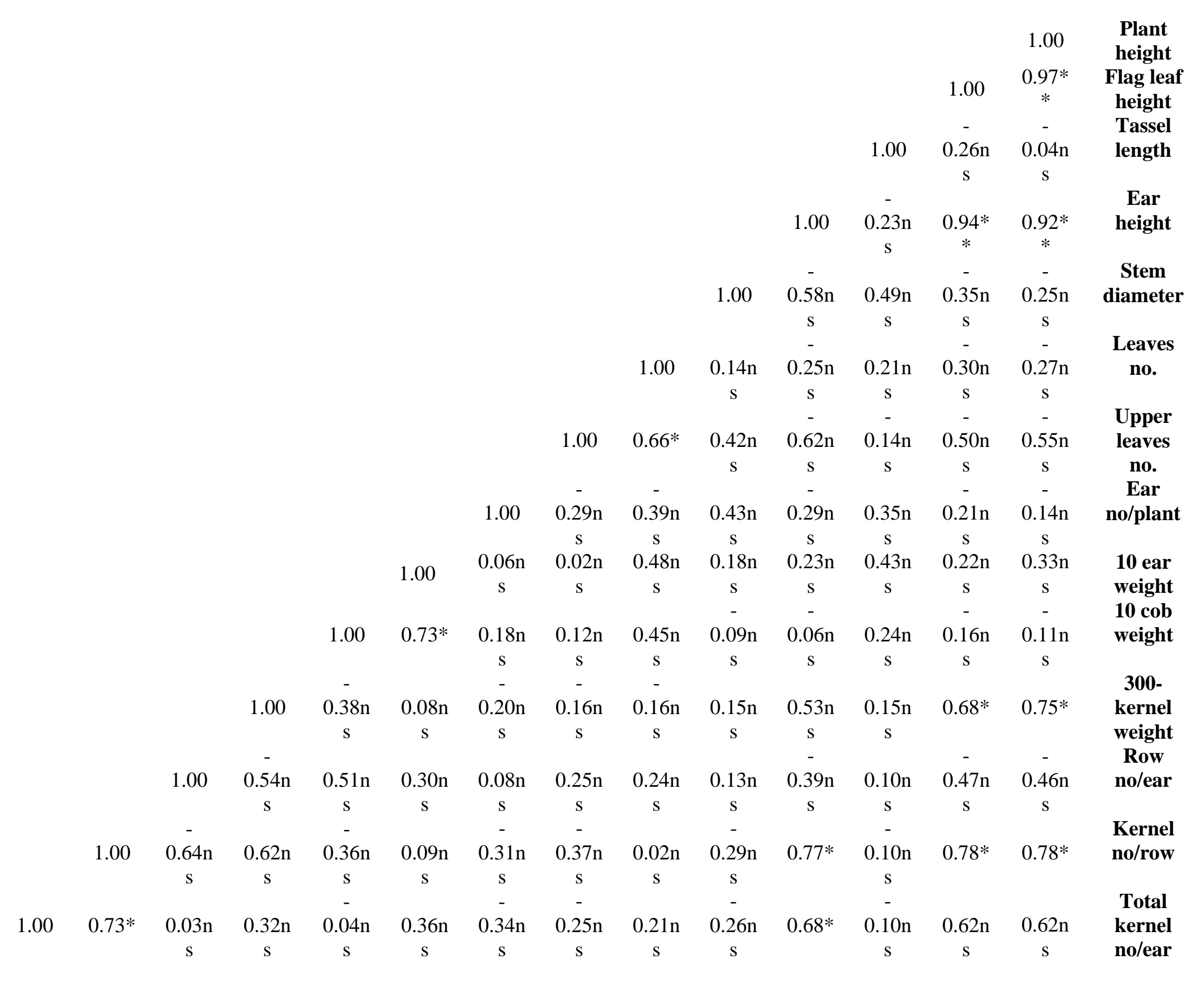




\begin{tabular}{|c|c|c|c|c|c|c|c|c|c|c|c|c|c|c|c|c|c|c|c|}
\hline & & & & 1.00 & $\begin{array}{c}0.34 \mathrm{n} \\
\mathrm{s}\end{array}$ & $\begin{array}{c}0.63 n \\
s\end{array}$ & $\begin{array}{c}- \\
0.51 \mathrm{n} \\
\mathrm{s}\end{array}$ & $\begin{array}{c}0.32 n \\
s\end{array}$ & $\begin{array}{c}0.22 n \\
s\end{array}$ & $\begin{array}{c}0.47 \mathrm{n} \\
\mathrm{s}\end{array}$ & $\begin{array}{c}0.09 n \\
s\end{array}$ & $\begin{array}{c}0.60 \mathrm{n} \\
\mathrm{s}\end{array}$ & $\begin{array}{c}0.01 \mathrm{n} \\
\mathrm{s}\end{array}$ & $\begin{array}{c}0.35 n \\
s\end{array}$ & $0.67 *$ & $\begin{array}{c}0.30 n \\
s\end{array}$ & $\begin{array}{c}0.58 \mathrm{n} \\
\mathrm{s}\end{array}$ & $0.68^{*}$ & $\begin{array}{l}\text { Ear } \\
\text { length }\end{array}$ \\
\hline & & & 1.00 & $\begin{array}{c}- \\
0.39 n \\
s\end{array}$ & $\begin{array}{c}- \\
0.02 \mathrm{n} \\
\mathrm{s}\end{array}$ & $\begin{array}{c}- \\
0.51 \mathrm{n} \\
\mathrm{s}\end{array}$ & $0.75^{*}$ & $\begin{array}{c}- \\
0.43 n \\
s\end{array}$ & $0.73^{*}$ & $\begin{array}{c}0.52 n \\
s\end{array}$ & $\begin{array}{c}0.02 n \\
s\end{array}$ & $0.62 *$ & $\begin{array}{c}0.60 n \\
s\end{array}$ & $\begin{array}{c}0.22 \mathrm{n} \\
\mathrm{s}\end{array}$ & $\begin{array}{c}- \\
0.38 \mathrm{n} \\
\mathrm{s}\end{array}$ & $\begin{array}{c}- \\
0.02 n \\
s\end{array}$ & $\begin{array}{c}- \\
0.38 \mathrm{n} \\
\mathrm{s}\end{array}$ & $\begin{array}{c}0.40 n \\
s\end{array}$ & $\begin{array}{c}\text { Ear } \\
\text { diameter }\end{array}$ \\
\hline & & 1.00 & $0.79 *$ & $\begin{array}{c}- \\
0.12 \mathrm{n} \\
\mathrm{s}\end{array}$ & $\begin{array}{c}0.23 n \\
s\end{array}$ & $\begin{array}{c}- \\
0.55 n \\
s\end{array}$ & $\begin{array}{c}0.58 \mathrm{n} \\
\mathrm{s}\end{array}$ & $\begin{array}{c}- \\
0.58 \mathrm{n} \\
\mathrm{s}\end{array}$ & $\begin{array}{l}0.84^{*} \\
*\end{array}$ & $\begin{array}{c}0.41 \mathrm{n} \\
\mathrm{s}\end{array}$ & $\begin{array}{c}- \\
0.14 \mathrm{n} \\
\mathrm{s}\end{array}$ & $\begin{array}{c}0.46 n \\
s\end{array}$ & $\begin{array}{c}0.58 n \\
s\end{array}$ & $\begin{array}{c}- \\
0.18 \mathrm{n} \\
\mathrm{s}\end{array}$ & $\begin{array}{c}- \\
0.32 \mathrm{n} \\
\mathrm{s}\end{array}$ & $\begin{array}{c}- \\
0.01 \mathrm{n} \\
\mathrm{s}\end{array}$ & $\begin{array}{c}- \\
0.45 n \\
s\end{array}$ & $\begin{array}{c}0.46 n \\
s\end{array}$ & $\begin{array}{c}\text { Cob } \\
\text { diameter }\end{array}$ \\
\hline & 1.00 & $\begin{array}{c}- \\
0.14 \mathrm{n} \\
\mathrm{s}\end{array}$ & $\begin{array}{c}0.48 \mathrm{n} \\
\mathrm{s}\end{array}$ & $\begin{array}{c}- \\
0.45 \mathrm{n} \\
\mathrm{s}\end{array}$ & $\begin{array}{c}0.34 n \\
s\end{array}$ & $\begin{array}{c}- \\
0.02 \mathrm{n} \\
\mathrm{s}\end{array}$ & $\begin{array}{c}0.40 \mathrm{n} \\
\mathrm{s}\end{array}$ & $\begin{array}{c}0.14 n \\
s\end{array}$ & $\begin{array}{c}- \\
0.02 \mathrm{n} \\
\mathrm{s}\end{array}$ & $\begin{array}{c}0.25 n \\
s\end{array}$ & $\begin{array}{c}0.25 n \\
s\end{array}$ & $\begin{array}{c}0.35 n \\
s\end{array}$ & $\begin{array}{c}0.13 n \\
s\end{array}$ & $\begin{array}{c}0.63 n \\
s\end{array}$ & $\begin{array}{c}- \\
0.16 \mathrm{n} \\
\mathrm{s}\end{array}$ & $\begin{array}{c}- \\
0.01 \mathrm{n} \\
\mathrm{s}\end{array}$ & $\begin{array}{c}0.02 n \\
s\end{array}$ & $\begin{array}{c}0.02 \mathrm{n} \\
\mathrm{s}\end{array}$ & $\begin{array}{l}\text { Kernel } \\
\text { depth }\end{array}$ \\
\hline 1.00 & $\begin{array}{c}0.21 \mathrm{n} \\
\mathrm{s}\end{array}$ & $\begin{array}{c}- \\
0.86^{*} \\
*\end{array}$ & $\begin{array}{c}- \\
0.63 n \\
s\end{array}$ & $\begin{array}{c}0.10 \mathrm{n} \\
\mathrm{s}\end{array}$ & $\begin{array}{c}0.37 n \\
s\end{array}$ & $\begin{array}{c}0.64 n \\
s\end{array}$ & $\begin{array}{c}- \\
0.56 \mathrm{n} \\
\mathrm{s}\end{array}$ & $0.69 *$ & $\begin{array}{c}- \\
0.82^{*} \\
*\end{array}$ & $\begin{array}{c}0.23 n \\
s\end{array}$ & $\begin{array}{c}- \\
0.26 n \\
s\end{array}$ & $\begin{array}{c}- \\
0.14 \mathrm{n} \\
\mathrm{s}\end{array}$ & $\begin{array}{c}- \\
0.27 \mathrm{n} \\
\mathrm{s}\end{array}$ & $\begin{array}{c}0.22 n \\
s\end{array}$ & $\begin{array}{c}0.36 n \\
s\end{array}$ & $\begin{array}{c}- \\
0.07 \mathrm{n} \\
\mathrm{s}\end{array}$ & $\begin{array}{c}0.52 n \\
s\end{array}$ & $\begin{array}{c}0.52 \mathrm{n} \\
\mathrm{s}\end{array}$ & $\begin{array}{c}\text { Kernel } \\
\text { percentag } \\
\text { e }\end{array}$ \\
\hline $\begin{array}{c}0.61 \mathrm{n} \\
\mathrm{s}\end{array}$ & $\begin{array}{c}0.39 n \\
s\end{array}$ & $\begin{array}{c}- \\
0.62 \mathrm{n} \\
\mathrm{s}\end{array}$ & $\begin{array}{c}- \\
0.31 \mathrm{n} \\
\mathrm{s}\end{array}$ & $\begin{array}{c}0.42 n \\
s\end{array}$ & $0.73^{*}$ & $0.65^{*}$ & $\begin{array}{c}- \\
0.35 n \\
s\end{array}$ & $\begin{array}{c}0.83^{*} \\
*\end{array}$ & $\begin{array}{c}- \\
0.23 n \\
s\end{array}$ & $\begin{array}{c}0.28 n \\
s\end{array}$ & $\begin{array}{c}0.05 n \\
s\end{array}$ & $\begin{array}{c}- \\
0.38 \mathrm{n} \\
\mathrm{s}\end{array}$ & $\begin{array}{c}- \\
0.16 n \\
s\end{array}$ & $\begin{array}{c}0.13 n \\
s\end{array}$ & $\begin{array}{c}0.66 n \\
s\end{array}$ & $\begin{array}{l}0.19 n \\
s\end{array}$ & $0.77^{*}$ & $\begin{array}{c}0.84 * \\
*\end{array}$ & $\begin{array}{l}\text { Total } \\
\text { yield }\end{array}$ \\
\hline
\end{tabular}




\begin{tabular}{|c|c|c|c|c|c|c|c|c|c|c|c|c|c|c|c|c|c|c|c|}
\hline & & & & & & & & & & & & & & & & & & & Trait \\
\hline & & & & & & & & & & & & & & & & & & 1.00 & Plant height \\
\hline & & & & & & & & & & & & & & & & & 1.00 & $0.94^{* *}$ & $\begin{array}{c}\text { Flag leaf } \\
\text { height }\end{array}$ \\
\hline & & & & & & & & & & & & & & & & 1.00 & $\begin{array}{c}- \\
0.09 \mathrm{~ns}\end{array}$ & $0.23 \mathrm{~ns}$ & Tassel length \\
\hline & & & & & & & & & & & & & & & 1.00 & - & $0.91 * *$ & $0.81^{* *}$ & Ear height \\
\hline & & & & & & & & & & & & & & 1.00 & $0.15 n s$ & $0.51 \mathrm{~ns}$ & $\begin{array}{c}- \\
0.05 \mathrm{~ns}\end{array}$ & $0.11 \mathrm{~ns}$ & Stem diameter \\
\hline & & & & & & & & & & & & & 1.00 & $0.38 \mathrm{~ns}$ & $0.33 \mathrm{~ns}$ & $\begin{array}{c}- \\
0.12 \mathrm{~ns}\end{array}$ & $0.18 \mathrm{~ns}$ & $0.22 \mathrm{~ns}$ & Leaves no. \\
\hline & & & & & & & & & & & & 1.00 & $0.58 \mathrm{~ns}$ & $0.39 \mathrm{~ns}$ & $0.44 \mathrm{~ns}$ & - & $0.26 \mathrm{~ns}$ & $0.22 \mathrm{~ns}$ & $\begin{array}{l}\text { Upper leaves } \\
\text { no. }\end{array}$ \\
\hline & & & & & & & & & & & 1.00 & $-0.85^{* *}$ & $\begin{array}{c}- \\
0.54 \mathrm{~ns}\end{array}$ & $-0.17 n s$ & $-0.53 \mathrm{~ns}$ & $0.29 \mathrm{~ns}$ & - & $\begin{array}{c}- \\
0.30 \mathrm{~ns}\end{array}$ & Ear no/plant \\
\hline & & & & & & & & & & 1.00 & $\begin{array}{c}- \\
0.06 \mathrm{~ns}\end{array}$ & $-0.14 \mathrm{~ns}$ & $0.33 \mathrm{~ns}$ & $-0.60 \mathrm{~ns}$ & $0.10 \mathrm{~ns}$ & $\begin{array}{c}- \\
0.38 \mathrm{~ns}\end{array}$ & $0.18 \mathrm{~ns}$ & $0.05 \mathrm{~ns}$ & 10 ear weight \\
\hline & & & & & & & & & 1.00 & $0.58 \mathrm{~ns}$ & $\begin{array}{c}- \\
0.11 \mathrm{~ns}\end{array}$ & $0.15 \mathrm{~ns}$ & $0.61 \mathrm{~ns}$ & $0.004 \mathrm{~ns}$ & $0.47 \mathrm{~ns}$ & $\begin{array}{c}- \\
0.30 \mathrm{~ns}\end{array}$ & $0.34 \mathrm{~ns}$ & $0.23 \mathrm{~ns}$ & 10 cob weight \\
\hline & & & & & & & & 1.00 & $\begin{array}{c}- \\
0.02 \mathrm{~ns}\end{array}$ & $0.29 \mathrm{~ns}$ & $0.14 \mathrm{~ns}$ & $-0.08 \mathrm{~ns}$ & $0.44 \mathrm{~ns}$ & $0.07 \mathrm{~ns}$ & $-0.34 n s$ & $0.67 *$ & $\begin{array}{c}- \\
0.21 \mathrm{~ns}\end{array}$ & $0.01 \mathrm{~ns}$ & $\begin{array}{l}\text { 300-kernel } \\
\text { weight }\end{array}$ \\
\hline & & & & & & & 1.00 & $\begin{array}{c}- \\
0.16 \mathrm{~ns}\end{array}$ & $0.17 \mathrm{~ns}$ & $0.74^{*}$ & $\begin{array}{c}- \\
0.32 \mathrm{~ns}\end{array}$ & $\begin{array}{c}- \\
0.005 \mathrm{~ns}\end{array}$ & $\begin{array}{c}- \\
0.03 \mathrm{~ns}\end{array}$ & $-0.68^{*}$ & $0.10 \mathrm{~ns}$ & $-0.70^{*}$ & $0.15 \mathrm{~ns}$ & $\begin{array}{c}- \\
0.07 \mathrm{~ns}\end{array}$ & Row no/ear \\
\hline & & & & & & 1.00 & $0.89 * *$ & $\begin{array}{c}- \\
0.14 \mathrm{~ns}\end{array}$ & $0.10 \mathrm{~ns}$ & $0.71^{*}$ & $0.09 \mathrm{~ns}$ & $-0.30 \mathrm{~ns}$ & $\begin{array}{c}- \\
0.33 \mathrm{~ns}\end{array}$ & $-0.81^{* *}$ & $-0.10 \mathrm{~ns}$ & $\begin{array}{c}- \\
0.60 \mathrm{~ns}\end{array}$ & $0.01 \mathrm{~ns}$ & $\begin{array}{c}- \\
0.18 \mathrm{~ns}\end{array}$ & Kernel no/row \\
\hline & & & & & 1.00 & $0.97 * *$ & $0.95^{* *}$ & $0.19 \mathrm{~ns}$ & $0.11 \mathrm{~ns}$ & $0.71^{*}$ & $0.10 \mathrm{~ns}$ & $-0.14 \mathrm{~ns}$ & $0.23 \mathrm{~ns}$ & $-0.76^{*}$ & $0.002 \mathrm{~ns}$ & $-0.66^{*}$ & $0.09 \mathrm{~ns}$ & $\begin{array}{c}- \\
0.12 \mathrm{~ns}\end{array}$ & $\begin{array}{l}\text { Total kernel } \\
\text { no/ear }\end{array}$ \\
\hline & & & & 1.00 & $0.51 \mathrm{~ns}$ & $0.58 \mathrm{~ns}$ & $0.51 \mathrm{~ns}$ & $0.13 \mathrm{~ns}$ & $0.16 \mathrm{~ns}$ & $0.65 \mathrm{~ns}$ & $0.35 \mathrm{~ns}$ & $-0.73^{*}$ & $0.18 \mathrm{~ns}$ & $-0.58 \mathrm{~ns}$ & $-0.04 \mathrm{~ns}$ & $\begin{array}{c}- \\
0.21 \mathrm{~ns}\end{array}$ & $0.10 \mathrm{~ns}$ & $0.03 \mathrm{~ns}$ & Ear length \\
\hline & & & 1.00 & $\begin{array}{c}- \\
0.50 \mathrm{~ns}\end{array}$ & $\begin{array}{c}- \\
0.30 \mathrm{~ns}\end{array}$ & $\begin{array}{c}- \\
0.28 \mathrm{~ns}\end{array}$ & $\begin{array}{c}- \\
0.44 \mathrm{~ns}\end{array}$ & $\begin{array}{c}- \\
0.06 \mathrm{~ns}\end{array}$ & $\begin{array}{c}- \\
0.19 \mathrm{~ns}\end{array}$ & $0.48 \mathrm{~ns}$ & $0.15 \mathrm{~ns}$ & $0.11 \mathrm{~ns}$ & $0.22 \mathrm{~ns}$ & $0.39 \mathrm{~ns}$ & $0.03 \mathrm{~ns}$ & $0.53 \mathrm{~ns}$ & $0.16 \mathrm{~ns}$ & $0.33 \mathrm{~ns}$ & Ear diameter \\
\hline & & 1.00 & $0.89 * *$ & $\begin{array}{c}- \\
0.65 \mathrm{~ns}\end{array}$ & $\begin{array}{c}- \\
0.37 \mathrm{~ns}\end{array}$ & $\begin{array}{c}- \\
0.39 \mathrm{~ns}\end{array}$ & $\begin{array}{c}- \\
0.45 \mathrm{~ns}\end{array}$ & $\begin{array}{c}- \\
0.28 \mathrm{~ns}\end{array}$ & $\begin{array}{c}- \\
0.01 \mathrm{~ns}\end{array}$ & $0.55 \mathrm{~ns}$ & $\begin{array}{c}- \\
0.06 \mathrm{~ns}\end{array}$ & $0.34 \mathrm{~ns}$ & $0.04 \mathrm{~ns}$ & $0.64 \mathrm{~ns}$ & $0.22 \mathrm{~ns}$ & $0.32 \mathrm{~ns}$ & $0.17 \mathrm{~ns}$ & $0.28 \mathrm{~ns}$ & Cob diameter \\
\hline & 1.00 & $\overline{-}$ & $0.02 \mathrm{~ns}$ & $0.51 \mathrm{~ns}$ & $0.06 \mathrm{~ns}$ & $0.10 \mathrm{~ns}$ & $0.06 \mathrm{~ns}$ & $0.64 \mathrm{~ns}$ & $\overline{-}-\overline{\mathrm{n}} \mathrm{ns}$ & $0.19 \mathrm{~ns}$ & $0.23 \mathrm{~ns}$ & $-0.46 \mathrm{~ns}$ & $\begin{array}{c}- \\
0.11 \mathrm{~ns}\end{array}$ & $-0.23 \mathrm{~ns}$ & $-0.31 \mathrm{~ns}$ & $0.55 \mathrm{~ns}$ & $0.01 \mathrm{~ns}$ & $0.19 \mathrm{~ns}$ & Kernel depth \\
\hline 1.00 & $0.58 \mathrm{~ns}$ & $\begin{array}{c}- \\
0.62\end{array}$ & $\begin{array}{c}- \\
0.35 \mathrm{~ns}\end{array}$ & $0.48 \mathrm{~ns}$ & $0.67 *$ & $0.69 *$ & $0.63 \mathrm{~ns}$ & $0.31 \mathrm{~ns}$ & $\overline{-}$ & $0.45 \mathrm{~ns}$ & $\begin{array}{c}- \\
0.09 \mathrm{~ns}\end{array}$ & $-0.32 \mathrm{~ns}$ & $\begin{array}{c}- \\
0.33 n s\end{array}$ & $-0.72^{*}$ & $-0.48 \mathrm{~ns}$ & $0.15 \mathrm{~ns}$ & $\begin{array}{c}- \\
0.27 \mathrm{~ns}\end{array}$ & $\begin{array}{c}- \\
0.31 \mathrm{~ns}\end{array}$ & $\begin{array}{c}\text { Kernel } \\
\text { percentage }\end{array}$ \\
\hline $0.62 \mathrm{~ns}$ & $0.40 \mathrm{~ns}$ & $\begin{array}{c}- \\
0.37\end{array}$ & $\begin{array}{c}- \\
0.13 \mathrm{~ns}\end{array}$ & $0.69 *$ & $0.63 \mathrm{~ns}$ & $0.81^{* *}$ & $0.47 \mathrm{~ns}$ & $0.29 \mathrm{~ns}$ & $0.08 \mathrm{~ns}$ & $0.64 \mathrm{~ns}$ & $0.76^{*}$ & $-0.68 *$ & $\begin{array}{c}- \\
0.33 \mathrm{~ns}\end{array}$ & $0.62 \mathrm{~ns}$ & $-0.38 \mathrm{~ns}$ & $\begin{array}{c}- \\
0.07 \mathrm{~ns}\end{array}$ & $\begin{array}{c}- \\
0.20 \mathrm{~ns}\end{array}$ & $\begin{array}{c}- \\
0.22 \mathrm{~ns}\end{array}$ & Total yield \\
\hline
\end{tabular}


Kernel yield values in normal irrigation and drought condition were presented in table 4. According to Fernandez (1992) the best measure for selection in drought condition could be able to separate genotypes which have desirable and similar yield in stress and non-tress condition from other groups and also, the best indices are those which have high correlation with kernel yield in both conditions. $\mathrm{H} 8$ and $\mathrm{H} 4$ with SSI (0.53 and 0.76 values) had the lowest susceptibility and H3 and H6 with SSI (1.32 and 1.11 values) had the highest susceptibility to drought pressure (table 4). More GOL indicates that yield value in drought pressure is close to yield potential and the studied hybrid was damaged lesser. Based on GOL index, H4, H5 and H8 had more resistance to drought. To evaluate hybrids with using TOL index, higher value of TOL demonstrates more changes of hybrids yield in stress and non-stress conditions and shows the susceptibility to non-stress condition. Fernandez (1992) and Rosielli and Hamblin (1981) stated that selection based on TOL index leads to selection of genotypes which their yields in non-stress condition are low and have lower MP. The results of this experiment showed that $\mathrm{H} 8$ (3.25) and $\mathrm{H} 6$ (8.77) were the most tolerant and sensitive hybrids based on TOL index to the drought pressure (table 4).

Table 4: Values of drought tolerance different indices

\begin{tabular}{|c|c|c|c|c|c|c|c|c|c|}
\hline Hybrid & GOL & HARM & STI & GMP & MP & TOL & SSI & $\mathbf{Y}_{\mathrm{S}}$ & $\overline{Y_{P}}$ \\
\hline 1 & 1.99 & 6.15 & 0.41 & 7.10 & 8.21 & 8.23 & 1.09 & 4.09 & 12.33 \\
\hline 2 & 2.01 & 5.06 & 0.28 & 5.83 & 6.71 & 6.66 & 1.08 & 3.38 & 10.04 \\
\hline 3 & 1.47 & 3.34 & 0.17 & 4.55 & 6.20 & 8.42 & 1.32 & 1.99 & 10.42 \\
\hline 4 & 3.28 & 7.12 & 0.46 & 7.47 & 7.84 & 4.77 & 0.76 & 5.46 & 10.23 \\
\hline 5 & 2.39 & 6.03 & 0.36 & 6.63 & 7.30 & 6.09 & 0.96 & 4.25 & 10.35 \\
\hline 6 & 1.93 & 6.19 & 0.43 & 7.24 & 8.46 & 8.77 & 1.11 & 4.08 & 12.85 \\
\hline 7 & 2.16 & 5.54 & 0.32 & 6.25 & 7.05 & 6.51 & 1.03 & 3.79 & 10.31 \\
\hline 8 & 5.15 & 8.07 & 0.55 & 8.22 & 8.38 & 3.25 & 0.53 & 6.75 & 10.01 \\
\hline KSC704 & 2.15 & 6.73 & 0.47 & 7.60 & 8.58 & 7.95 & 1.03 & 4.60 & 12.56 \\
\hline
\end{tabular}

In order to investigate the relationship between indices simple phenotypic correlation analysis was done and the results were presented in table 5. Maximum positive correlation was observed between GMP and STI (1) and maximum negative correlation was recorded between SSI and yield in drought condition (0.95). MP index directs breeders to select in stress and non-stress condition. KSC704 hybrid (8.58) and H3 (6.20) were the most tolerant and sensitive hybrids to pressure (table 4). According to the Fernandez (1992) model, STI index is calculated based on yields geometric mean productivity (GMP) in both conditions. Mathematical basis of this index has been being designed that whether far differences between yield means of stress and non-stress condition, 
GMP tends to the low value and has efficiency in selection of tolerant hybrids to drought. H8 (8.22) and H3 (4.55) were the most tolerant and sensitive hybrids to water shortage based on GMP (table 4). High value of STI indicated more tolerance of genotypes to drought. Regarding hybrids kernel yield, H8 (0.55) and KSC704 (0.47) revealed maximum tolerance to drought (table 4). Furthermore, HARM index demonstrated the advantage of $\mathrm{H} 8$ than others to drought tension (8.07) and $\mathrm{H} 3$ has the minimum tolerance to drought stress by this index. Correlation between drought tolerance indices and yield can be used as a suitable measure for best hybrids selection. Yahoueian et al., (2005) announced that GMP and Fernandez index are paramount indices in evaluate soybean hybrids in drought pressure. Mehrabi et al., (2011) corn hybrids with high yield can be obtained based on GMP and STI indices. Jafari et al., (2009) found that STI, GMP and HARM indices which showed the highest correlation with grain yield under both optimal and stress conditions, can be used as the best indices for maize breeding programs to introduce drought tolerant hybrids. Moghaddam and Hadizadeh (2000) and Ahmadzadeh (1997) claimed that MP index is much better than SSI and TOL in selection of drought tolerant genotypes. Correlation results showed positive and significant relationship between potential yield (non-stress condition) with STI, GMP, MP, HARM and TOL, while correlation between yield and TOL was negative and significant $\left(\mathrm{r}=0.76^{* *}\right)$ in stress condition (table 5). In this study, since MP, GMP, HARM and Fernandez index had high, positive and significant correlation with yield in normal irrigation and drought pressure, are introduced as the superior indices. Fereres et al., (1983) in study of genotypes reaction to drought focused on yield sensitivity to drought. 
Table 5: Phenotypic correlation coefficients of drought tolerance under stress and non-stress condition

\begin{tabular}{rrrrrrrrr}
\hline & $\mathbf{Y}_{\mathbf{P}}$ & $\mathbf{Y}_{\mathbf{S}}$ & SSI & TOL & MP & GMP & STI & MHAR \\
\hline $\mathbf{Y}_{\mathbf{P}}$ & 1 & & & & & & & \\
$\mathbf{Y} \mathbf{S}$ & $-0.07 \mathrm{~ns}$ & 1 & & & & & & \\
$\mathbf{S S I}$ & $0.35 \mathrm{~ns}$ & $-0.95^{* *}$ & 1 & & & & & \\
$\mathbf{T O L}$ & $0.69^{*}$ & $-0.76^{*}$ & $0.91^{* *}$ & 1 & & & & \\
$\mathbf{M P}$ & $0.87^{* *}$ & $0.71^{*}$ & $-0.48 \mathrm{~ns}$ & $-0.10 \mathrm{~ns}$ & 1 & & & \\
$\mathbf{G M P}$ & $0.83^{* *}$ & $0.91^{* *}$ & $-0.76^{*}$ & $-0.48 \mathrm{~ns}$ & $0.92^{* *}$ & 1 & & \\
$\mathbf{S T I}$ & $0.83^{* *}$ & $0.91^{* *}$ & $-0.76^{*}$ & $-0.48 \mathrm{~ns}$ & $0.92^{* *}$ & 1 & 1 & \\
$\mathbf{M H A R}$ & $0.76^{*}$ & $0.97 * *$ & $-0.87 * *$ & $-0.62 \mathrm{~ns}$ & $0.83^{* *}$ & $0.98^{* *}$ & $0.98^{* *}$ & \\
$\mathbf{G O L}$ & $-0.39 \mathrm{~ns}$ & $0.90^{* *}$ & $-0.96^{* *}$ & $-0.90^{* *}$ & $0.42 \mathrm{~ns}$ & $0.67 *$ & $0.71^{*}$ & $0.78^{*}$ \\
\hline
\end{tabular}

Table 6: Selected hybrids based on drought tolerance different indices

\begin{tabular}{|c|c|}
\hline Selected hybrids (right to left) & Index \\
\hline 3 ، KSC704 ، 6 & $Y_{P}$ \\
\hline KSC704 ، 4 & $\mathrm{Y}_{\mathrm{S}}$ \\
\hline $5 \quad 4 \quad 48$ & SSI \\
\hline $5 \quad 6 \quad 8$ & TOL \\
\hline $8 \cdot 6 \cdot \mathrm{KSC} 704$ & MP \\
\hline $4 \cdot \mathrm{KSC704} \cdot 8$ & GMP \\
\hline 4 ، KSC704 ، 8 & STI \\
\hline $\mathrm{KSC704} \cdot 4 \cdot 8$ & MHAR \\
\hline $\begin{array}{lll}5 & 6\end{array}$ & GOL \\
\hline
\end{tabular}

\section{References}

Ahamadzade, A. 1997. The determination of the best drought tolerance indices in corn elite lines. M.Sc. Thesis. Faculty of Agriculture, The university of Tehran. Pp. 90-150.

Alahdadi I, Oraki H, Parhizkar khajani F (2011) Effect of water stress on yield and yield components of sunflower hybrids. Afr J Biotechnol 10(34): 6504-6509.

Available online at:

http://www.academicjournals.org/ajb/PDF/pdf2011/11Jul/Alahdadi $\% 20$ et $\% 20$ al.pdf 
Ali, Q., M. Elahi. M. Ahsan. M. H. Nadeem Tahir. S. M. A. Basra. 2011. Genetic evaluation of maize (zea maize L.) genotypes at seedling stage under moisture stress. IJAVMS 5 (2): 184-193.

Available online at:

http://www.scopemed.org/mnstemps/25/25-1306091840.pdf

Bolanos, J., and G. O. Edemedes.1996. The importance of the anthesis-silking interval inbreeding for drought. Tolerance in tropical maize. Field Crops. Res. 48:65-80.

Dash, B., S.V. Singh, and J.P. Shahi.1999.Character association and path analysis in s1 lines of maize.J.Agric. Res.5: 14-32.

Eck, H. V. 1984. Irrigated corn yield response to nitrogen and water. Agron J 76: 421428.

Available online at:

http://ddr.nal.usda.gov/bitstream/10113/502/1/IND84055663.pdf

Egilla, J. N., Jr. F. T. Davies. T. W. Boutton. 2005. Drought stress influences leaf water content, photosynthesis, and water-use efficiency of Hibiscus rosasinensis at three potassium concentrations. Photosynth, 43: 135-140.

Available online at:

http://essm.tamu.edu/people/boutton/WUE\%20and\%20potassium\%20-\%20Egilla.pdf

Esmailiyan, Y., M. Heidari. A. Ghanbari. 2008. Effect of municipal wastewater with manure and chemical fertilizer on grain yield and yield components in corn KoSc 704. J Agron.

Available online at:

http://www.docsdrive.com/pdfs/ansinet/ja/0000/2764-2764.pdf

Farre, I., M. V. Oijen. P. A. Leffelaar. J. M. Faci. 2000. Analysis of Maize growth for different irrigation strategies in northeastern Spain. European J. of Agron. 12: 225-238. Available online at:

http://directory.umm.ac.id/Data\%20Elmu/jurnal/E/European\%20Journal\%20of\%20 Agronomy/Vol12.Issue3-4.Jun2000/7.pdf

Fereres, E., Gimenz, C., Brenngena, J., Fernandez, J., and Domiguez, J. 1983. Genetic variability of sunflower cultivar in response to drought. Helia 6: 17-21.

Fernandez, G. C. 1992. Effective selection criteria for assessing plant stress tolerance. In: Proceedings of the Symposium of AVRDC, 13-16 Aug. Taiwan.

Fisher, R. A. R. Maurer.1978. Drought resistance in spring wheat cultivars. I. Grain yield responses. Australian J of Agric Res 29: 897-912. 
Golbashy, M., M. Ebrahimi. S. Khavari Khorasani. R. Choukan. 2010. Evaluation of drought tolerance of some corn (Zea mays L.) hybrids in Iran. Afr J Agric Res 5(19): 2714-2719.

Available online at:

http://www.academicjournals.org/ajar/PDF/pdf $\% 202010 / 4 \% 20$ Oct/Golbashy $\% 20$ et \%20al.pdf

Hirich, A., A. Rami. K. Laajaj. R. Choukr-Allah. S-E. Jacobsen. L. El youssfi. H. Omari. 2012. Sweet corn water productivity under several deficit irrigation regimes applied during vegetative growth stage using treated wastewater as water irrigation source. World Academy of Science, Engineering and Technology 61.

Available online at: http://www.waset.org/journals/waset/v61/v61-156.pdf

Jafari, A., F. Paknejad. M. Jami Al-Ahmadi. 2009. Evaluation of selection indices for drought tolerance of corn (Zea mays L.) hybrids. Inter J of Plant Prod 3 (4).

Available online at:

http://www.gau.ac.ir/Jm/Programs/JurnalMgr/VolumArticle/EN 139 5.pdf

Khazaei, F., M. Agha Alikhani. L. Yari. A. Khandan. 2010. Study the correlation, regression and path coefficient analysis in sweet corn (Zea mays var. saccharata) under different levels of plant density and nitrogen rate. ARPN J of Agric and Biol Sci 5(6).

Available online at:

http://www.arpnjournals.com/jabs/research papers/rp 2010/jabs 1110 217.pdf

Khodarahmpour, Z. 2011. Effect of drought stress induced by polyethylene glycol (PEG) on germination indices in corn (Zea mays L.) hybrids. Afr J Biotechnol 10 (79): 18222-18227.

Available online at:

http://www.academicjournals.org/ajb/PDF/pdf2011/12Dec/Khodarahmpour.pdf

Lorense, G. F., Bennett, J. M. and Loggale, L. B. 1987. Differences in drought resistance between two corn hybrids. II. Component analysis and growth rates. Agronomy Journal 79: 808-813.

Magorokosho, C., K. V. Pixley. P. Tongoona. 2003. Selection for drought tolerance in two tropical maize populations. Afr Crop Science J 11(3): 151-161.

Available online at: http://www.bioline.org.br/pdf?cs03018

Mehrabi, P., H. Homayoun. M. S. Daliri. 2011. Study of drought tolerance of corn genotypes using STI index. Middle-East J Sci Res 9 (1): 68-70.

Available online at: http://www.idosi.org/mejsr/mejsr9(1)11/11.pdf 
Messmer, R. E. 2006. The genetic dissection of key factors involved in the drought tolerance of tropical maize (zea maize L.). A dissertation submitted to the Swiss Federal Institute of Technology Zurich.

Available online at:

http://e-collection.library.ethz.ch/eserv/eth:29035/eth-29035-01.pdf

Moghaddam, A, and M. H. Hadizadeh. 2002. Response of corn (Zea mays L.) hybrids and their parental lines to drought using different stress tolerance indices. 18(3): 255-272.

Moradi Dezfuli, P., F. Sharif-zadeh. M. Janmohammadi. 2008. Influence of priming techniques on seed germination behavior of maize inbred lines (Zea mays L.). ARPN J of Agric and Biol Sci. 3 (3):

Available online at:

http://www.arpnjournals.com/jabs/research papers/rp 2008/jabs 0508 79.pdf

Mostafavi, Kh., M. Shoahosseini. H. Sadeghi Geive. 2011. Multivariate analysis of variation among traits of corn hybrids traits under drought stress. Inter J AgriSci 1(7): 416-422.

Available online at:

http://www.inaci.com/attachments/section/17/2011-

284\%20Mostafavi $\% 20$ and $\% 20$ ShoaHosseini $\% 20 \mathrm{E} \% 20$ CLEAN $\% 20 \mathrm{~F} \% 20 \mathrm{P} \% 20(\mathrm{pp} \% 20416$ -

422)\%202.pdf

Oktem, A. 2008. Effects of deficit irrigation on some yield characteristics of sweet corn. Bangladesh J Bot 37(2): 127-131.

Available online at:

http://www.banglajol.info/index.php/BJB/article/download/1718/1630

Oraki, H., I. Alahdadi. F. Parhizkar khajani. 2011. Investigation of the effect of water deficit stress on yield and yield components in sunflower hybrids. Afr J Agric Res 6(10): 2358-2363.

Available online at:

http://www.academicjournals.org/ajar/PDF/pdf2011/18\%20May/Oraki\%20et\%20al.p df

Ouattar, S., Jones, R. J. and Crookstone, R. K. 1987. Effect of water deficit during grain filling on the pattern of maize kernel growth and development. Crop Science 27: 726730.

Palta, C., U. Karadavut. M. Tezel. S. Aksoyak. 2011. Agronomic performance of some corn cultivars (Zea maize L.) in middle Anatolia. J Anim Vet Adv 10(14): 1901-1905.

Available online at:

http://docsdrive.com/pdfs/medwelljournals/javaa/2011/1901-1905.pdf 
Payero, J. O., S. R. Melvin. S. Irmak. D. Tarkalson. 2006. Yield response of corn to deficit irrigation in a semiarid climate. Agricultural Water Management 84(1-2): 101-112. Doi: $10.1016 /$ j.agwat.2006.01.009

Rosielli, A., J. Hamblin. 1981. Theoritical aspects of selection for yield in stress and nonstress environment. Crop Sci 21: 493.

Sinaki, M. J., E. Majidi Heravan. H. Shiranirad. G. Noormohammadi. G. H. Zarei. 2007. The effects of water deficit during growth stages of canola (Brassica napus L.). AmEuras. J. Agric. Environ. Sci. 2: 417-422.

Available online at: http://www.idosi.org/aejaes/jaes2(4)/17.pdf

Song, Y., C. Qu. S. Birch. A. Doherty. J. Hanan. 2010. Analysis and modelling of the effects of water stress on maize growth and yield in dryland conditions. Plant Prod Sci 13(2): 199-208.

Available online at:

http://www.jstage.jst.go.jp/article/pps/13/2/199/_pdf

Vaezi, B., A. Ahmadikhah. 2010. Evaluation of drought tolerance of twelve improved barley genotypes in dry and warm condition. J of Plant Production 17(1). (In Persian) Available online at:

http://www.sid.ir/fa/VEWSSID/J pdf/37613890102.pdf

Yahoueian, S. H., Ghannadha, M. R., Babaie, H. R., and Habibi, D. 2006.

Evaluation of soybean genotypes in drought stress conditions. Iranian Journal of Agronomy and Plant Breeding 2 (2): 57-72 (in Farsi).

Zarabi, M., I. Alahdadi. G. A. Akbari. G. A. Akbari. 2011. A study on the effects of different biofertilizer combinations on yield, its components and growth indices of corn (Zea mays L.) under drought stress condition. Afr J Agric Res 6(3): 681-685.

Available online at:

http://www.academicjournals.org/ajar/pdf/pdf2011/4\%20Feb/Zarabi $\% 20$ et $\% 20$ al.pdf 
